\title{
Preparation and Evaluation of the Antibacterial Effect of Magnetic Nanoparticles Containing Gentamicin: A Preliminary In vitro Study
}

\author{
Banafsheh Douzandeh-Mobarrez ${ }^{1}$, Mehdi Ansari-Dogaheh ${ }^{2}$, Touba Eslaminejad ${ }^{2}$, Maryam Kazemipour ${ }^{3}$, \\ Mojtaba Shakibaie 2,4,"
}

${ }^{1}$ Herbal and Traditional Medicines Research Center, Kerman University of Medical Sciences, Kerman, Iran
${ }^{2}$ Pharmaceutics Research Center, Institute of Neuropharmacology, Kerman University of Medical Sciences, Kerman, Iran
${ }^{3}$ Department of Chemistry, Faculty of Sciences, Kerman Branch, Islamic Azad University, Kerman, Iran
${ }^{4}$ Department of Pharmaceutical Biotechnology, Faculty of Pharmacy, Kerman University of Medical Sciences, Kerman, Iran

"Corresponding author: Mojtaba Shakibaie, Pharmaceutics Research Center, Institute of Neuropharmacology, Kerman University of Medical Sciences, Kerman, Iran. Tel.: +98 3431325253, Fax: +98 3431325003. E-mail: shakiba@kmu.ac.ir

Received:25 Apr. 2016; $\quad$ Revised: 4 Aug. 2018; $\quad$ Accepted: 29 Aug. 2018; $\quad$ Published online: 12 Dec. 2018

\begin{abstract}
Background: Magnetic nanoparticles (MNPs) loaded by various active compounds can be used for targeted drug delivery. Objectives: In the present study, the $\mathrm{Fe}_{3} \mathrm{O}_{4}$ magnetic nanoparticles that contained gentamicin were prepared and their antibacterial activities were studied.

Materials and Methods: MNPs containing gentamicin (G@SA-MNPs) were prepared using sodium alginate (SA) as a surface modifier. After and before coating, the prepared MNPs were characterized using transmission electron microscopy (TEM), X-ray diffraction spectroscopy (XRD), Fourier transform infrared spectroscopy (FTIR), and vibrating sample magnetometer (VSM). Finally, the antibacterial effect of the MNPs was investigated by a conventional serial agar dilution method.

Results: Particle size distribution analysis showed that the size of MNPs, before and after coating, was in the range of 1-18 $\mathrm{nm}$ and 12-40 nm, respectively. The magnetization curve of G@SA-MNPs (with saturation magnetization of 27.9 emu.g ${ }^{-1}$ ) confirmed ferromagnetic property. Loading gentamicin on the surface of MNPs was qualitatively verified by FTIR spectrum. Quantitative analysis measurements indicated the gentamicin loading on SA-MNPs as 56.7 $\pm 5.4 \%$. The measured MICs of G@SA-MNPs for Pseudomonas aeruginosa (PTTC 1574) was $1.28 \mu \mathrm{g} . \mathrm{mL}^{-1}$. The sub-MIC $\left(0.64 \mu \mathrm{g} \cdot \mathrm{mL}^{-1}\right)$ concentration of G@SA-MNPs in nutrient broth could successfully inhibit the growth of $P$. aeruginosa for 14 hours.

Conclusions: Loading gentamicin on the SA-MNPs exhibited reasonable antibacterial effects against $P$. aeruginosa.

Keywords: Antibacterial activities; $\mathrm{Fe}_{3} \mathrm{O}_{4}$; Gentamicin; Magnetic nanoparticles
\end{abstract}

\section{Background}

Drug targeting, as a promising tool to increase the activity and decrease the side effects of drugs, has received significant attention in recent years (1). Targeted drug delivery can be applied effectively for controlling microorganisms and preventing harmful infections (2). In addition, application of nanoparticles in therapeutic or diagnostic agents has received much attention (3). The next generation of nanoparticle-based research is directed at multifunctional systems, which could facilitate the individual therapy (4).

Among nanomaterials used in drug delivery systems, magnetic nanoparticles (MNPs) received more application, especially in targeted drug delivery systems (5). When MNPs are exposed to an external magnetic field with various inductions and gradients, they can be transported to a certain location and act as effective drug carriers (6). MNPs exhibited large

Copyright (C) 2018 The Author(s); Published by National Institute of Genetic Engineering and Biotechnology. This is an open access article, distributed under the terms of the Creative Commons Attribution-NonCommercial 4.0 International License (http://creativecommons.org/licenses/ by-nc/4.0/) which permits others to copy and redistribute material just in noncommercial usages, provided the original work is properly cited. 
surface to volume ratio and they can adsorb significant amounts of active compounds using various polymers $(7,8)$. Loading different compounds such as anti-cancer and antibacterial compounds on MNPs could serve as a novel strategy for targeted therapy and the inhibition of drug resistance $(9,10)$. In this regard, several methods have been reported to make use of different magnetic NPs (11).

Gentamicin is an aminoglycoside used to treat many types of bacterial infections caused by gramnegative and gram-positive bacteria (12). Kidney damage and ear disorders are the most important side effect of gentamicin. Different bacterial resistance mechanisms to gentamicin have been reported as well (12). Preparations of different gentamicin-containing formulations, such as liposomes (13) and microspheres (14), were previously reported. Furthermore, the antibacterial effects of different nanostructures containing gentamicin, such as controlled-release and poly(lactide-co-glycolide) nanoparticles $(15,16)$, and chitosan/fucoidan nanoparticles (17), have been confirmed.

\section{Objectives}

Bearing in mind the aforementioned points about MNPs and antimicrobial activity of gentamicin, the aim of the present study was to produce MNPs coated with gentamicin. In addition, the antibacterial effect of the prepared MNPs was investigated.

\section{Materials and Methods}

\subsection{Materials and Microorganisms}

Nutrient broth (NB), Muller-Hinton broth (MHB), Agar, Sodium alginate (SA), Ferrous ammonium sulfate $\left(\left(\mathrm{NH}_{4}\right)_{2} \mathrm{Fe}\left(\mathrm{SO}_{4}\right)_{2} \cdot 6 \mathrm{H}_{2} \mathrm{O}\right)$, and Ferric ammonium sulfate $\left(\mathrm{NH}_{4} \mathrm{Fe}\left(\mathrm{SO}_{4}\right)_{2} 12 \mathrm{H}_{2} \mathrm{O}\right)$ were purchased from Merck Chemicals (Darmstadt, Germany). Gentamicin sulfate (G) was prepared from Sina Daru pharmaceutical company (Tehran, Iran). Ninhydrin was purchased from Suvchem Laboratory Chemicals (Suvchem, India) and deionized water was prepared by Milli-Q water (Millipore, USA). All the other chemicals and solvents were of analytical grade. The following representative strain of Pseudomonas aeruginosa (PTTC 1574) was purchased from the Persian type culture collection (Tehran, Iran). In order to obtain fresh active cultures, bacteria were sub-cultured on the Mueller-Hinton agar plate and incubated at $37^{\circ} \mathrm{C}$ for $24 \mathrm{~h}$.

\subsection{Preparation of Sodium Alginate Modified MNPS (SA-MNPS)}

$\mathrm{SA}$ modified magnetic $\mathrm{Fe}_{3} \mathrm{O}_{4}$ nanoparticles was prepared by a co-precipitation method via alkalization of an aqueous solution containing $\mathrm{Fe}^{2+}$ and $\mathrm{Fe}^{3+}$ ions (6). In brief, ferrous ammonium sulfate $(0.425 \mathrm{~g})$ and ferric ammonium sulfate $(0.625 \mathrm{~g})$ were separately dissolved in $100 \mathrm{~mL}$ deionized water, which was deoxygenated by bubbling nitrogen gas $(1 \mathrm{~h})$. After mixing the solutions by a magnetic stirrer $(500 \mathrm{rpm}$, $3 \mathrm{~min}$ ), $10 \mathrm{~mL}$ of Ammonia solution (25\%) was added in drops to the aforementioned mixture as it was being stirred with mechanical agitation $\left(1000 \mathrm{rpm}, 40{ }^{\circ} \mathrm{C}\right)$ until the mixture turned dark. The black precipitate of the $\mathrm{Fe}_{3} \mathrm{O}_{4}$ NPs was washed three times with deionized water and MNPs were suspended as a $1 \mathrm{mg} \cdot \mathrm{mL}^{-1}$ mixture. To prepare SA-MNPs, $6.25 \mathrm{~mL}$ of SA solution $(0.01 \%)$ was stirred with $12.5 \mathrm{~mL}$ of the MNPs mixture $\left(24 \mathrm{~h}, 4{ }^{\circ} \mathrm{C}\right)$. After centrifugation $(2000 \times g, 10 \mathrm{~min})$, the supernatant (including free SA) was discarded and the prepared SA-MNPs were lyophilized using a freeze dryer (FD-81; Eyela, Tokyo, Japan) and stored in a sealed container at $4{ }^{\circ} \mathrm{C}$.

\subsection{Preparation of Gentamicin Loaded on SA-MNPS (G@SA-MNPs)}

To load gentamicin to the surface of the prepared SA-MNPs, a simple precipitation method based on the positive charges of gentamicin and the negative charge of SA-MNPs was used. To summarize, SAMNPs suspension was prepared by dispersing 100 $\mathrm{mg}$ of SA-MNPs in $100 \mathrm{~mL}$ of deionized water using ultrasonication ( $100 \mathrm{~W}, 5 \mathrm{~min})$. Subsequently, 6.25 $\mathrm{mL}$ of aqueous solution of $\mathrm{G}\left(1 \mathrm{mg} \cdot \mathrm{mL}^{-1}\right)$ was added in drops to $18.75 \mathrm{~mL}$ of the prepared SA-MNPs suspension under continuous stirring using a laboratory magnetic stirrer (300 rpm, 2 h). The resulting G@SAMNPs was separated from the aqueous media using a magnet, and the supernatant was stored for detecting the free gentamicin. Finally, the capped NPs were washed four times with deionized water using centrifugation $(14000 \times g, 10 \mathrm{~min})$ and, afterwards, was lyophilized and stored in a sealed container at $4{ }^{\circ} \mathrm{C}$.

\subsection{Characterizations of the MNPS}

The prepared NPs were characterized using various instruments before and after being loaded with gentamicin. Transmission electron micrographs of ultrasonicated NPs were obtained using TEM equipment (Zeiss 902A, South Jen Germany) operated at an accelerating voltage of $80 \mathrm{kV}$. The related size distribution pattern of NPs was plotted by manually counting 250 individual particles from different TEM images. X-ray diffraction (XRD) data of the synthesized NPs were collected on a P3000 diffractometer instrument 
(Rich Seifert, NY, USA) employing $\mathrm{Cu}-\mathrm{K} \alpha$ radiation at a voltage of $40 \mathrm{kV}$ and a current of $30 \mathrm{~mA}$. The magnetic properties of MNPs and G@SA-MNPs were determined using a vibrating sample magnetometer (Lakeshore, Westerville, OH, USA) with a maximum magnetic field of $10 \mathrm{kOe}$ at room temperature. The dried MNPs (before and after the loading), gentamicin, and SA used for Fourier transform infrared spectroscopy analysis by an FTIR instrument (Tensor 27, Bruker Optics, Germany) at a resolution of $4 \mathrm{~cm}^{-1}$ in $\mathrm{KBr}$ pellets. For quantitative analysis of the coating process, a spectrophotometric method with some modification was applied for measuring the un-loaded gentamicin (16). To summarize, $5 \mathrm{~mL}$ of different concentrations of gentamicin $\left(50-300 \mu \mathrm{g} . \mathrm{mL}^{-1}\right)$ were mixed with 1.5 $\mathrm{mL}$ of Ninhydrin aqueous solution $(1.25 \% \mathrm{w} / \mathrm{v})$ and $4.5 \mathrm{~mL}$ of PBS ( $\mathrm{pH} 7.4)$. The mixture was incubated in a water bath $\left(95^{\circ} \mathrm{C}, 15 \mathrm{~min}\right)$ and after cooling on an ice bath absorbencies were measured at $400 \mathrm{~nm}$ using a spectrophotometer (UV-2100, Shimadzu, Japan). These procedures were performed in triplicate on different days, and the mean of the obtained absorbencies was used to plot a suitable standard curve. The estimation of gentamicin uptake on the SA-MNPs was done by measuring the difference in gentamicin concentration in solution before and after loading. In all the absorption measurements, the mixture containing SA-MNPs was subjected to the same steps as the blank. The drug entrapment percentage was calculated according to the following equation:

Drug entrapment $(\%)=100 \times\left(C_{I}-C_{F}\right) / C_{I}$,

Where $C_{I}$ was the initial concentration of gentamicin, $\mathrm{C}_{\mathrm{F}}$ was concentration of gentamicin in the supernatant. The drug loading percent as the amount of drug per unit weight percent of the drug delivery systems is calculated using the following equation:

Drug loading $(\%)=($ Entrapped Drug/nanoparticles weight) $\times 100$

G@SA-MNPs was prepared on different days and the mean of drug loading (\%) was measured.

\subsection{Antibacterial Activity of MNPS}

The agar dilution method, which involves the incorporation of different concentrations of the antimicrobial substance into an agar-containing culture medium (18) was used for determining the minimal inhibitory concentration (MIC) of gentamicin (G), G@ SA-MNPs, and SA-MNPs. Briefly, Mueller-Hinton agar plates were prepared with a twofold dilution series of gentamicin $\left(0.25 \mu \mathrm{g} \cdot \mathrm{mL}^{-1}\right.$ to $\left.16 \mu \mathrm{g} \cdot \mathrm{mL}^{-1}\right), \mathrm{G} @$ SA-MNPs $\left(0.08 \mu \mathrm{g} \cdot \mathrm{mL}^{-1}\right.$ to $\left.10.24 \mu \mathrm{g} \cdot \mathrm{mL}^{-1}\right)$, and SAMNPs $\left(5 \mu \mathrm{g} \cdot \mathrm{mL}^{-1}\right.$ to $\left.640 \mu \mathrm{g} \cdot \mathrm{mL}^{-1}\right)$. Then, $10^{4}$ colonyforming units of the overnight culture of Pseudomonas aeruginosa (PTTC 1574) were separately transferred to mentioned plates and plates were incubated for 24 hours at $37^{\circ} \mathrm{C}$. The aforementioned method was performed in triplicate on different days and the MIC was recorded as the lowest concentration of agent inhibited visible growth of the bacteria.

In order to investigate the growth trajectory of bacteria in the presence of MNPs, P. aeruginosa was selected and subMIC concentration of gentamicin, G@SA-MNPs, and SAMNPs were separately prepared by $50 \mathrm{~mL}$ sterile nutrient broth media in $250 \mathrm{~mL}$ Erlenmeyer flasks. Afterward, fresh inoculum $\left(1 \mathrm{~mL}, \mathrm{OD}_{600} 0.1\right)$ of $P$. aeruginosa was transferred to above flasks. ( $\left.37{ }^{\circ} \mathrm{C}, 160 \mathrm{rpm}\right)$. Culture media containing gentamicin, G@SA-MNPs, and SAMNPs without culturing by $P$. aeruginosa were prepared as blank. Next, flasks were plugged with cotton and incubated in a shaking incubator $\left(37^{\circ} \mathrm{C}, 160 \mathrm{rpm}\right)$, and $\mathrm{OD}_{600} \mathrm{~nm}$ was measured with a spectrophotometer (UV2100, Shimadzu, Japan). Four replications were run for each treatment and the process was repeated three times.

\subsection{Statistical Analysis}

SPSS version 15.0 for Microsoft Windows (SPSS, Chicago, IL, USA) was used for statistical analysis. For paired observation, differences were determined using the paired $t$-test and $P$ values of less than 0.05 were considered to be statistically significant.

\section{Results}

Before and after the coating process with SA and gentamicin, the shape and size properties of the synthesized magnetic NPs were studied by TEM. The TEM images of MNPs and G@SA-MNPs are presented in Figure 1a and 1b, respectively. G@SA-MNPs were dispersed into single particles with round surface shape, small size, and a slight aggregation, while naked MNPs particles exhibited greater aggregations and turned into clusters of particles (Fig. 1b). The size distribution patterns revealed that the MNPs and G@SA-MNPs were in the range between $1 \mathrm{~nm}$ to $18 \mathrm{~nm}$ and $12 \mathrm{~nm}$ to $40 \mathrm{~nm}$, respectively (Fig. 1c). Figure 2a exhibited the related XRD pattern of G@SA-MNPs with seven characteristic peaks of $2 \theta, 30.1^{\circ}, 35.6^{\circ}, 43.3^{\circ}, 53.5^{\circ}, 57^{\circ}$, $63^{\circ}$, and $74^{\circ}$. No differences were observed in XRD patterns of MNPs before and after the coating process (data not shown). The hysteresis curves of MNPs before and after adding SA and gentamicin show that 

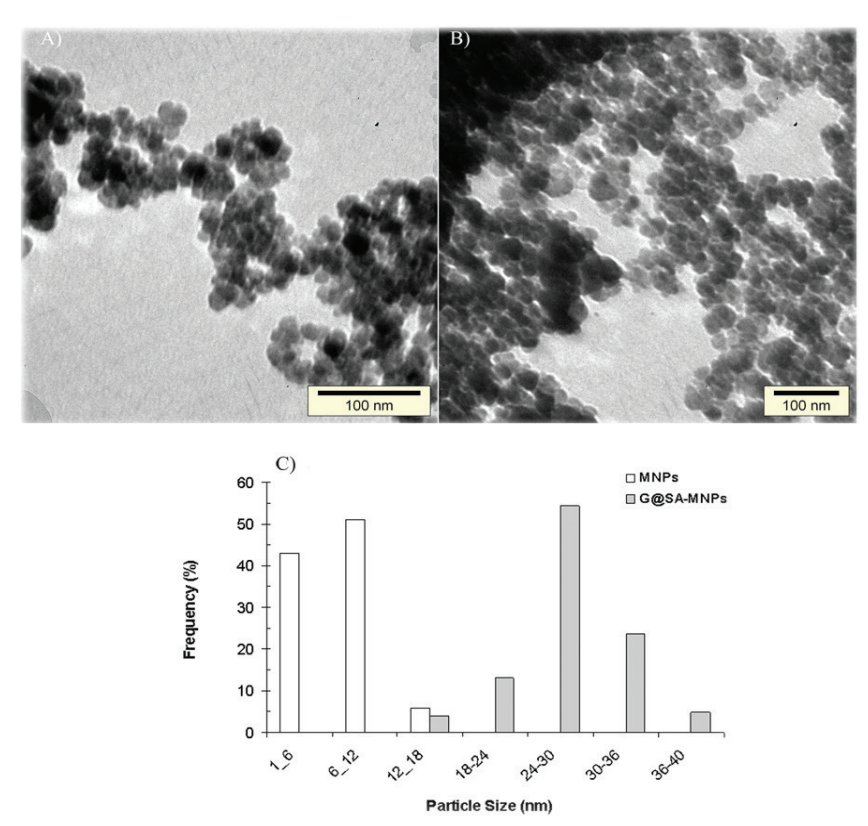

Figure 1. TEM micrographs of synthesized (A) $\mathrm{Fe}_{3} \mathrm{O}_{4}$ magnetic nanoparticles (MNPs), (B) Gentamicin-loaded MNPs modified with sodium alginate (G@SA-MNPs), and (C) particle size distribution patterns of $\mathrm{Fe}_{3} \mathrm{O}_{4}$ MNPs before and after the coating process with $\mathrm{SA}$ and gentamicin.
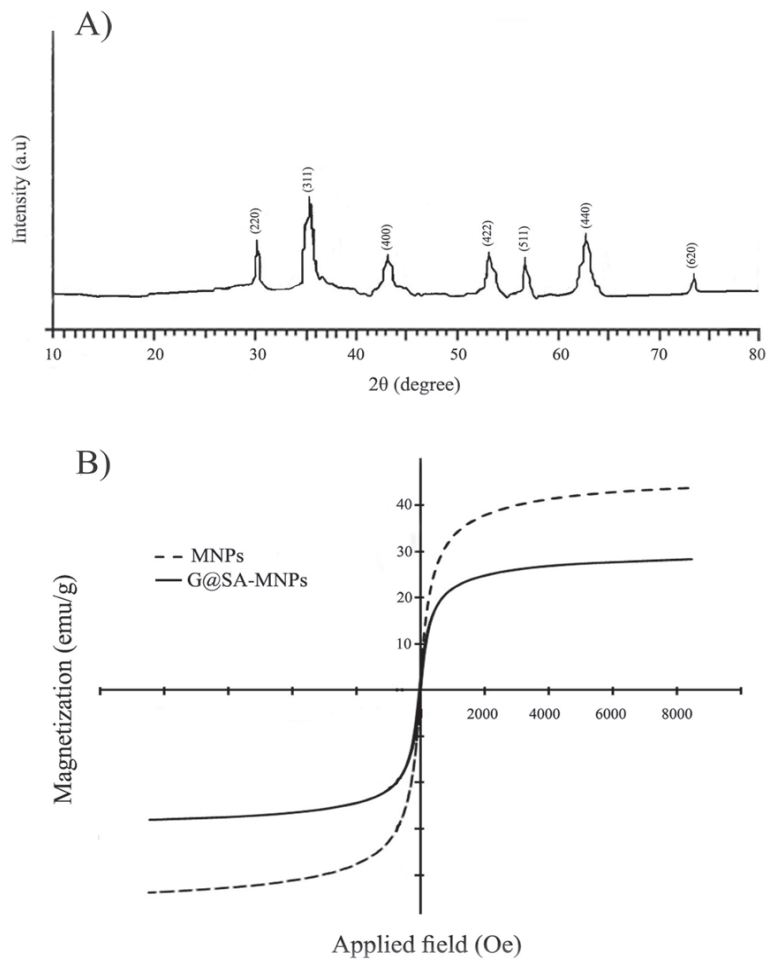

Figure 2. (A) X-ray diffraction pattern of Gentamicinloaded-sodium-alginate-modified MNPs (G@SA-MNPs), and (B) magnetic hysteresis curves of naked $\mathrm{Fe}_{3} \mathrm{O}_{4}$ MNPs and Gentamicin-loaded MNPs modified with sodium alginate (G@SA-MNPs). both samples have ferromagnetic properties (Fig. 2b). For qualitative analysis of the loading process, FTIR spectra of SA, SA-MNPs, G, MNPs, and G@SAMNPs are presented in Figures 3a to e, respectively. No significant differences were observed between FTIR spectra of the SA-MNPs (Fig. 3b), MNPs (Fig. 3d), and G@SA-MNPs (Fig. 3e) in different synthesis times. The amount of loaded gentamicin was detected by colorimetric method using UV-spectrophotometer. The standard curve exhibited suitable linearity between the gentamicin concentration and absorbance obtained at $400 \mathrm{~nm}$ and the correlation was similar to previous reports. The quantitative analysis exhibited that the gentamicin entrapment and the loading efficiency was $56.7 \pm 5.4 \%, 15.9 \pm 1.3 \%$, respectively.

MICs of gentamicin, SA-MNPs, and fabricated G@SA-MNPs were measured by a conventional serial agar dilution method. SA-MNPs did not show any antibacterial activity against the tested bacteria at a concentration range of 5-640 $\mu \mathrm{g} \cdot \mathrm{mL}^{-1}$. For $P$. aeruginosa (PTTC 1574) treated with gentamicin, the MIC was found to be $16 \mu \mathrm{g} \cdot \mathrm{mL}^{-1}$. However, in the case of G@SA-MNPs, MIC was determined to be $1.28 \mu \mathrm{g} . \mathrm{mL}^{-1}$. The results of a time-kill course study carried out with G@SA-MNPs against $P$. aeruginosa

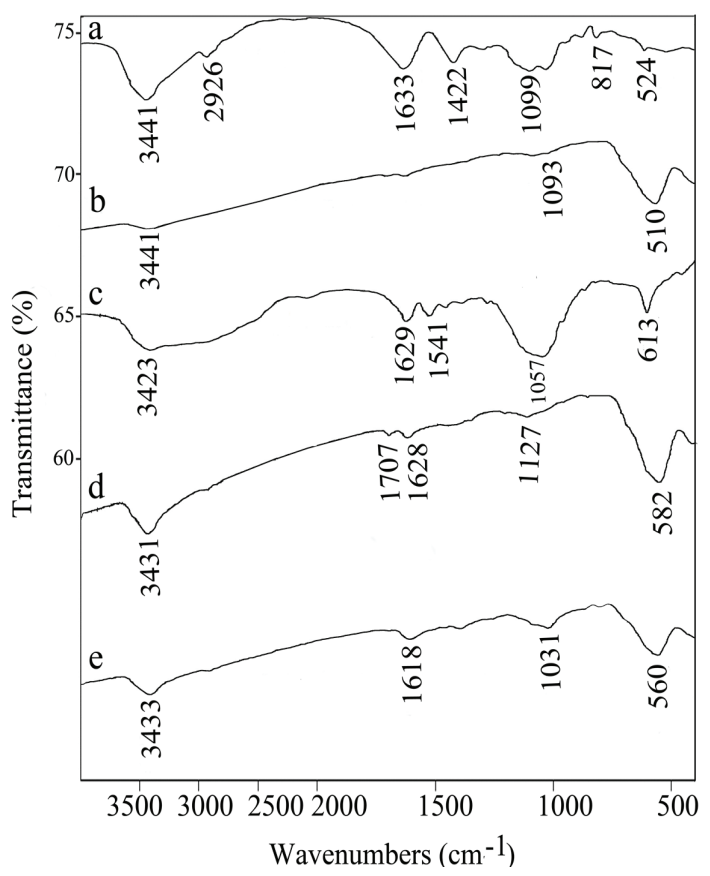

Figure 3. FTIR spectra of (A) Sodium alginate (SA), (B) $\mathrm{Fe}_{3} \mathrm{O}_{4}$ magnetic nanoparticles modified with sodium alginate (SA-MNPs) (C) Gentamicin sulfate (G), (D) naked $\mathrm{Fe}_{3} \mathrm{O}_{4}$ magnetic nanoparticles (MNPs), and (E) Gentamicin-loaded MNPs modified with sodium alginate (G@SA-MNPs). 
at sub-MIC concentrations $\left(0.64 \mu \mathrm{g} . \mathrm{mL}^{-1}\right)$ are shown in Figure 4. In comparison to the controls, G@SA-MNPs could successfully inhibit the growth of $P$. aeruginosa for 14 hours.

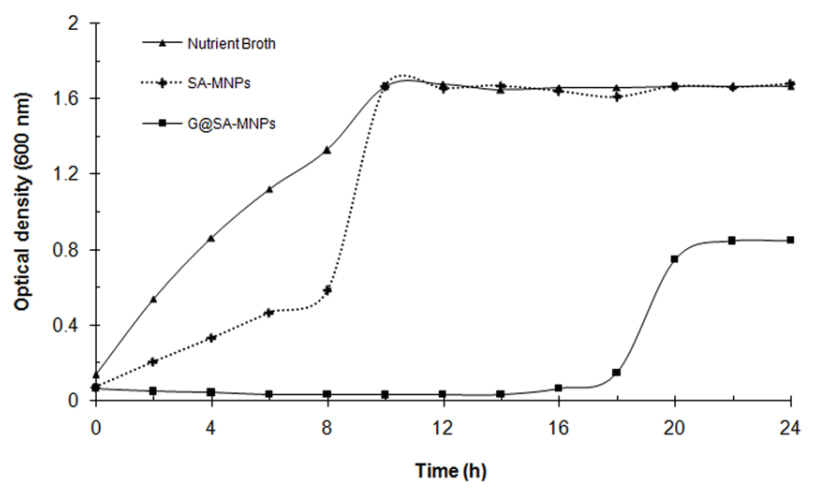

Figure 4. Growth patterns of $P$. aeruginosa (PTTC 1574) in the presence of sub-MIC concentration $\left(0.64 \mu \mathrm{g} . \mathrm{mL}^{-1}\right)$ of $\mathrm{Fe}_{3} \mathrm{O}_{4}$ magnetic nanoparticles modified with sodium alginate (SA-MNPs), and Gentamicin-loaded MNPs modified with sodium alginate (G@SA-MNPs).

\section{Discussion}

In this study, G@SA-MNPs was synthesized by chemical co-precipitation and the feasibility of these nanoparticles as an antimicrobial delivery system was studied. Co-precipitation is the most promising MNPs synthesis method because of its simplicity, productivity, and size controlling $(19,20)$. Aggregation between the NPs could be created in consequence of large surface to volume ratio and magnetic forces between the MNPs (21). The uncoated MNPs tended to quickly aggregate and precipitate after formation. Figure 1a demonstrates that there is an agglomeration of uncoated MNPs and they are aggregated into clusters of particles. Furthermore, the coating of MNPs with polymers is a common way to stabilize NPs against oxidation (22). The coated MNPs should be wellseparated due to the SA coating layer, but the clusters shown in Figure 1b can be explained by the drying process in the preparation for TEM measurements. Particles with the size of $6 \mathrm{~nm}$ to $12 \mathrm{~nm}$ and $24 \mathrm{~nm}$ to 30 nm were the most frequent particles in MNPs and G@ SA-MNPs, respectively (Fig. 1c). The results exhibited that the addition of gentamicin on the surface of the MNPs using SA significantly increased the size of the G@SA-MNPs compared with MNPs $(p<0.05)$. Such effects have been previously reported for MNPs coated with other compounds, such as piroctone olamine and umbelliprenin $(23,24)$. Furthermore, the surfaces of the MNPs were saturated with the adsorbed linear SA, and this induced both hydration forces and electrosteric stabilization due to the hydrophilic character of linear SA and the presence of charged polyelectrolyte loops, respectively.

The XRD pattern of MNPs (Fig. 2a) confirmed that the G@SA-MNPs was successfully synthesized and the coating process with SA and gentamicin did not have an obvious influence on the crystalline structure of $\mathrm{Fe}_{3} \mathrm{O}_{4}$ MNPs. The measured saturation magnetization (MS) for MNPs and G@SA-MNPs was 42.8 emu. $\mathrm{g}^{-1}$ and 27.9 emu. $\mathrm{g}^{-1}$, respectively (Fig. 2b). These values are clearly lower than the measured MS value for bulk $\mathrm{Fe}_{3} \mathrm{O}_{4}(90$ emu. $\left.\mathrm{g}^{-1}\right)$. Presence of SA and gentamicin on the surface of MNPs might account for a decrease in the MS of $\mathrm{G} @$ SA-MNPs. Such decrease in MS value of $\mathrm{Fe}_{3} \mathrm{O}_{4}$ nanoparticles modified by sodium citrate and oleic acid was previously reported by Wei et al. (25).

In the FT-IR spectrum of sodium alginate stretching vibrations of $\mathrm{O}-\mathrm{H}$ bonds of alginate appeared in the range of $3000-3600 \mathrm{~cm}^{-1}$ (Fig. 3a). The Stretching vibrations of aliphatic C-H were observed at 2920$2850 \mathrm{~cm}^{-1}$. Observed bands in $1633 \mathrm{~cm}^{-1}$ and 1422 $\mathrm{cm}^{-1}$ might be attributed to the asymmetric and the symmetric stretching vibrations of carboxylate salt ion, respectively (Fig. 3a). The FTIR spectra of gentamicin exhibited peaks at $1629 \mathrm{~cm}^{-1}$ and $1541 \mathrm{~cm}^{-1}$ correspond to amide band. Peaks at $613 \mathrm{~cm}^{-1}$ and $1057 \mathrm{~cm}^{-1}$ were due to the Sulphur in the form of S-O bending vibration and S-O stretching, respectively (Fig. 3c). The $\mathrm{Fe}_{3} \mathrm{O}_{4}$ MNPs exhibited the characteristic absorption of Fe-O and $\mathrm{O}-\mathrm{H}$ bonds at $582 \mathrm{~cm}^{-1}$, and $3431 \mathrm{~cm}^{-1}$, respectively (Fig. 3d). The characteristic bands of SA, gentamicin and naked MNPs were not observed in the spectrum of the G@SA-MNPs (Fig. 3e). In G@SA-MNPs spectrum, the typical band of gentamicin at $1541 \mathrm{~cm}^{-1}$ is cleared in the presence of SA; SA has a typical bond at $1633 \mathrm{~cm}^{-1}$ which is cleared in the presence of MNPs in G-SA-MNPs (Fig. 3e). The FT-IR study indicated the presence of SA and gentamicin on the G@SA-MNPs. Drug loading is an important factor affecting the trajectory and the amount of drug delivered to the target. In this study, the percentage of gentamicin loaded on the SA-MNPs was calculated as $56.7 \pm 5.4 \%$ and each 100 mg of the G@SA-MNPs contained 15.9 $\pm 1.3 \mathrm{mg}$ of gentamicin. Previously, Khorramizadeh et al. (24) reported that each milligram of prepared umbelliprenin-coated MNPs contained almost 250 $\mu \mathrm{g}$ of umbelliprenin. Shakibaie et al. reported the preparation of $\mathrm{Fe}_{3} \mathrm{O}_{4} @$ piroctone olamine MNPs (without using polymers), which each $1 \mathrm{mg}$ of the coated NPs contained $50 \pm 1.3 \mu \mathrm{g}$ of piroctone olamine (23). However, in the case of loading other antibiotics 
like amoxicillin on magnetic nanoparticles with Poly (lactide-co-glycolide) copolymer (PLGA) and Poly (lactide-co-glycolide)-polyethylene glycol copolymer (PLGA-PEG), the measured efficient entrapment was $90 \%$ and $48-52 \%$, respectively (26).

To study the interactions between G@SA-MNPs and bacterial strains, we took advantage of MIC technique. Comparison of the MIC values indicated that G@SA-MNPs could inhibit visible growth of all the tested bacteria at a lower concentration and it was relatively more effective than pure gentamicin at the same concentration. Results from turbidiometric assay (Fig. 4) revealed that the density of $P$. aeruginosa in media containing G@SA-MNPs was significantly reduced compared to NB and SA-MNPs $(p<0.05)$. Such effect has been previously reported for other gentamicin-loaded nanoparticles, which gradually release the antibiotic, by maintaining the stability and the effect of the antibiotic longer than soluble antibiotic (27). Furthermore, SA-MNPs exhibited some reduction in the growth of $P$. aeruginosa (Fig. 4). Earlier studies reported the significant increase in the antimicrobial propensity of iron oxide nanoparticles coated with other polymers like chitosan against Bacillus subtilis and Escherichia coli (28). It might be concluded that the antibiotic-coated MNPs maintain the antibacterial effect for the time longer than they are maintained for a free antibiotic.

\section{Conclusion}

In the present work, gentamicin entrapment and loading efficiency on magnetic nanoparticles was 56.7 $\pm 5.4 \%, 15.9 \pm 1.3 \%$, respectively and its antibacterial effect was evaluated. Gentamicin (as a positively charged molecule) was successfully conjugated with MNPs using sodium alginate (as a negatively charged polymer). To sum up, the prepared G@SA-MNPs has a good antibacterial activity against $P$. aeruginosa. Furthermore, loading of gentamicin on the SA-MNPs exhibited reasonable antibacterial effects. However, recycling and reusing of G@SA-MNPs based on their magnetic properties merit further investigation.

\section{Conflict of Interest}

The authors declare no conflict of interest.

\section{Acknowledgements}

This work was financially supported by Pharmaceutics Research Center, Kerman University of Medical Sciences (Kerman, Iran). We also thank the Iranian Nanotechnology Initiative Council for its admirable participation in this study.

\section{References}

1. Singh R, Lillard JW. Nanoparticle-based targeted drug delivery. Exp Mol Pathol. 2009;86:215-223. doi: 10.1016/j. yexmp.2008.12.004.

2. Zhang L, Pornpattananangkul D, Hu C-MJ, Huang C-M. Development of nanoparticles for antimicrobial drug delivery. Curr Med Chem. 2010;17:585-594. doi: 10.2174/092986710790416290.

3. Forootanfar H, Amirpour-Rostami S, Jafari M, Forootanfar A, Yousefizadeh Z, Shakibaie M. Microbial-assisted synthesis and evaluation the cytotoxic effect of tellurium nanorods. Mat Sci Eng C. 2015;49:183-189. doi: 10.1016/j.msec.2014.12.078.

4. Yu MK, Park J, Jon S. Targeting strategies for multifunctional nanoparticles in cancer imaging and therapy. Theranostics. 2012;2:3-44. doi: 10.7150/thno.3463.

5. Arruebo M, Fernández-Pacheco R. Magnetic nanoparticles for drug delivery. Nanotoday. 2007;2:22-32. doi: 10.1016/S17480132(07)70084-1.

6. Martínez-Mera I, Espinosa-Pesqueira ME, Pérez-Hernández R, Arenas-Alatorre J. Synthesis of magnetite $\left(\mathrm{Fe}_{3} \mathrm{O}_{4}\right)$ nanoparticles without surfactants at room temperature. Mater Lett. 2007;61:4447- 4451. doi: 10.1016/j.matlet.2007.02.018.

7. Berry CC, Curtis ASG. Functionalisation of magnetic nanoparticles for applications in biomedicine. J Phys D: Appl Phys. 2003;36:R198-R206. doi: 10.1088/0022-3727/36/13/203.

8. Kievit FM, Wang FY, Fang C, Mok H, Wang K, Silber JR, Ellenbogen RG, Zhang M. Doxorubicin loaded iron oxide nanoparticles overcome multidrug resistance in cancer in vitro. J Control Release. 2011;152:76-83. doi: 10.1016/j. jconrel.2011.01.024.

9. Dobretsov K, Stolyar S, Lopatin A. Magnetic nanoparticles: a new tool for antibiotic delivery to sinonasal tissues. Results of preliminary studies. Acta Otorhinolaryngol Ital. 2015;35:97102.

10. Jolivet JP, Cassaignon S, Chanéac C, Chiche D, Durupthy O, Portehault D. Design of metal oxide nanoparticles: Control of size, shape, crystalline structure and functionalization by aqueous chemistry. Comptes Rendus Chimie. 2010;13:40-1. doi: 10.1016/j.crci.2009.09.012.

11. Grumezescu M, Andronescu E, Holban AM, Ficai A, Ficai D, Voicu G, Grumezescu V, Balaure PC, Chifiriuc CM.Water dispersible cross-linked magnetic chitosan beads for increasing the antimicrobial efficiency of aminoglycoside antibiotics. Int $J$ Pharm. 2013;454:233-240. doi:10.1016/j.ijpharm.2013.06.054.

12. Jian X, Deng Z, Sun Y. Aminoglycoside gentamicin research: fundamental progress and new application prospects. Sheng Wu Gong Cheng Xue Bao. 2015;31:829-844. doi: 10.13345/j. cjb. 140635 .

13. Vitas AI, Diaz R, Gamazo C. Effect of composition and method of preparation of liposomes on their stability and interaction with murine monocytes infected with Brucella abortus. Antimicrob Agents Ch. 1996;40:146-151.

14. Prior S, Gander B, Lecaroz C, Irache JM, Gamazo C. Gentamicin loaded microspheres for reducing the intracellular Brucella abortus load in infected monocytes. J Antimicrob Chemoth. 2004;53:981-988. doi: 10.1093/jac/dkh227.

15. Lecaroz C, Blanco-Prieto MJ, Burrell MA, Gamazo C. Intracellular killing of Brucella melitensis in human macrophages with microsphere-encapsulated gentamicin. $J$ Antimicrob Chemoth. 2006;58:549-556. doi: 10.1093/jac/ dk1257. 


\section{Douzandeh-Mobarrez B et al.}

16. Dorati R, DeTrizio A, Genta I, Grisoli P, Merelli A, Tomasi C, Conti B. An experimental design approach to the preparation of pegylated polylactide-co-glicolide gentamicin loaded microparticles for local antibiotic delivery. Mat Sci Eng C. 2016;58:909-917. doi: 10.1016/j.msec.2015.09.053.

17. Huang YC, Li RY, Chen JY, Chen JK. Biphasic release of gentamicin from chitosan/fucoidan nanoparticles for pulmonary delivery. Carbohyd Polym.2016;138:114-122. doi: 10.1016/j. carbpol.2015.11.072.

18. Wiegand I, Hilpert K, Hancock REW. Agar and broth dilution methods to determine the minimal inhibitory concentration (MIC) of antimicrobial substances. Nat Protoc. 2008;3:163175. doi: 10.1038/nprot.2007.521.

19. Thach CV, Hai NH, Chau N. Size controlled magnetite nanoparticles and their drug loading ability. J Korean Phys Soc. 2008;52:1332-1335 . doi: 10.3938/jkps.52.1332.

20. Tresilwised N, Pithayanukul P, Plank C. Factors affecting sizes of magnetic particles formed by chemical co-precipitation. $J$ Pharm Sci. 2005;32:71-76.

21. Veyret, R, Delair T, Pichot C, Elaissari A. Amino-containing magnetic nanoemulsions: elaboration and nucleic acid extraction. J Magn Magn Mater. 2005;295:155-163. doi: 10.1016/j.jmmm.2005.01.008.

22. Shubayev VI, Pisanic TR, Jin S. Magnetic nanoparticles for theragnostics. Adv Drug Del Rev. 2009;61:467-477. doi: 10.1016/j.addr.2009.03.007.

23. Shakibaie M, Haghiri M, Jafari M, Amirpour-Rostami S, Ameri A, Forootanfar H, Mehrabani M. preparation and evaluation the effect of $\mathrm{Fe}_{3} \mathrm{O}_{4} @$ piroctone olamine magnetic nanoparticles on matrix metalloproteinase-2: a preliminary in vitro study. Biotechnol Appl Biochem. 2014;61:676-682. doi: 10.1002/ bab.1231.

24. Khorramizadeh MR, Esmail-Nazari Z, Zarei-Ghaane Z, Shakibaie M, Mollazadeh-Moghaddam K, Iranshahi M, Shahverdi AR. Umbelliprenin-coated $\mathrm{Fe}_{3} \mathrm{O}_{4}$ magnetite nanoparticles: Antiproliferation evaluation on human Fibrosarcoma cell line (HT-1080). Mat Sci Eng C. 2010;30:10381042. doi: 10.1016/j.msec.2010.05.005.

25. Wei Y, Han B, Hu X, Lin Y, Wang X, Deng X. Synthesis of $\mathrm{Fe}_{3} \mathrm{O}_{4}$ nanoparticles and their magnetic properties. Procedia Eng. 2012;27:632-637. doi: 10.1016/j.proeng.2011.12.498.

26. 26. Alimohammadi S, Salehi R, Amini N, Davaran S. Synthesis and physicochemical characterization of biodegradable PLGA-based magnetic nanoparticles containing amoxicilin. Bull Korean Chem Soc. 2012;33:3225-3232. doi: 10.5012/ bkcs.2012.33.10.3225.

27. Abdelghany SM, Quinn DJ, Gilmore BF, Donnelly RF, Taggart CC, Scott CJ. Gentamicin-loaded nanoparticles show improved antimicrobial effects towards Pseudomonas aeruginosa infection. Int J Nanomed.2012;7:4053-4063. doi: 10.2147/IJN. S34341.

28. Arakha M, Pal S, Samantarrai D, Panigrahi TK, Mallick BC, Pramanik K, Mallick B, Jha S. Antimicrobial activity of iron oxide nanoparticle upon modulation of nanoparticle-bacteria interface. Sci Rep. 2015;5:14813. doi: 10.1038/srep14813. 